\title{
Gravitational Wave sources in Ultralow frequency band
}

\author{
Avetis Abel Sadoyan \\ Yerevan State University \\ Alex Manoogian 1, Yerevan, 375070 Armenian \\ E-mail: asadoyan@ysu.am
}

Rainer Spurzem

Astronomical Rechen Institute, Heidelberg, Germany

and National Astronomical Observatory Beijing, China

E-mail: spurzemeari.uni-heidelberg.de

\section{Peter Berczik}

Astronomical Rechen Institute, Heidelberg, Germany and National Astronomical Observatory Beijing, China

E-mail: berczikeari.uni-heidelberg.de

\begin{abstract}
We had calculated possible gravitational wave emission from galaxies with supermasive double black holes in the center during their evolution simulated at Astronomical Rechen Institute in Heidelberg. Gravitational wave frequencies are calculated for the whole timescale of evolution, changing in the range from $10^{-13}$ to $10^{-10} \mathrm{~Hz}$, average amplitudes are around $10^{-22}$. The most promising results were obtained for ultrafast rotating galaxies, where velocities are close to Keplerian and where bar instabilities were formed. We show, that there are stages in the beginning of the evolution of the fast rotating galaxies, where the gravitational radiation from galaxy itself is competing, and may be even stronger than the gravitational radiation of binary black hole. We had calculated the total energy flux from the galaxy through gravitational radiation (Gravitational Luminosity) and had found large energy spikes in Gravitational Luminosity, while largest spikes are observed in the time period near to the end of evolution.
\end{abstract}

25th Texas Symposium on Relativistic Astrophysics -TEXAS2010

Heidelberg, Germany

December $06-112010$ 


\section{Introduction}

We had calculated possible gravitational wave emission during the evolution of galaxies simulated at Astronomical Rechnen Institute in Heidelberg. $\mathrm{N}$ body models were generated with phase-space distribution function according to [1] where the initial spherical model has a central concentration parameter $\mathrm{W}_{0}=6$ and a degree of flattening $\omega_{0}=1.8$ as in [2] . A pair of massive particles representing two black holes were introduced into the model at $\mathrm{t}=0$ with equal masses and were placed on coplanar circular orbits at distances \pm 0.3 in standard $\mathrm{N}$ body units, that is, the gravitational constant and total mass of galaxy are equal to 1 and the galaxy's energy is $-1 / 4$. Farther we are using $\mathrm{N}$ body unites. Integration of the particle equations of motion were carried out using a high-accuracy direct summation N-Body code [3] on two parallel supercomputers incorporating special purpose GRAPE[4]. Integration parameters were similar to those adopted in [3]. Integrations were carried out for various values of $\mathrm{N}$ in the range of $25 \times 10^{3}$ to $1 \times 10^{6}$. Detailed evolution of gravitational wave radiations is calculated in each case.

\section{Gravitational Radiation from Galaxies}

The purpose of this work is to calculate the possible gravitational wave radiation from galaxy itself and to compare the gravitational radiation from galaxy to gravitational radiation from supermassive black hole binary in the center.

Binary supermassive black holes are formed in galaxy mergers, but their long-term evolution is uncertain, in spherical galaxies, $\mathrm{N}$ body evolution shows that binary evolution stalls at separations much too large for significant emission of gravitational waves [5]. Unless the binary mass ratio is large, dynamical friction rapidly brings the binary into a separation of an order of $1 \mathrm{pc}$, when the central engine start to act like a "hard binary", ejecting passing stars with velocities large enough to leave the galaxy[6,5]. In the used simulations of galaxy evolution with super massive black holes a long term evolution of massive binary is followed in more realistic, triaxial and rotating galaxy models, where binary stalls only in initial phases of evolution, while later one can observe a binary hardening with rates sufficient to allow close coalescence approximately in 10 Gyear[5]. Our simulations were made up to $t=250$ in $\mathrm{N}$ body units which corresponds to $250 \times 10^{13} \mathrm{~s}$ or around 1 Gyear. We are concentrated in this work on gravitational radiation from galaxy itself, showing that the radiation from galaxy is 
not neglectable and can be registered in the scope of Parks Pulsar Timing Array project[9].
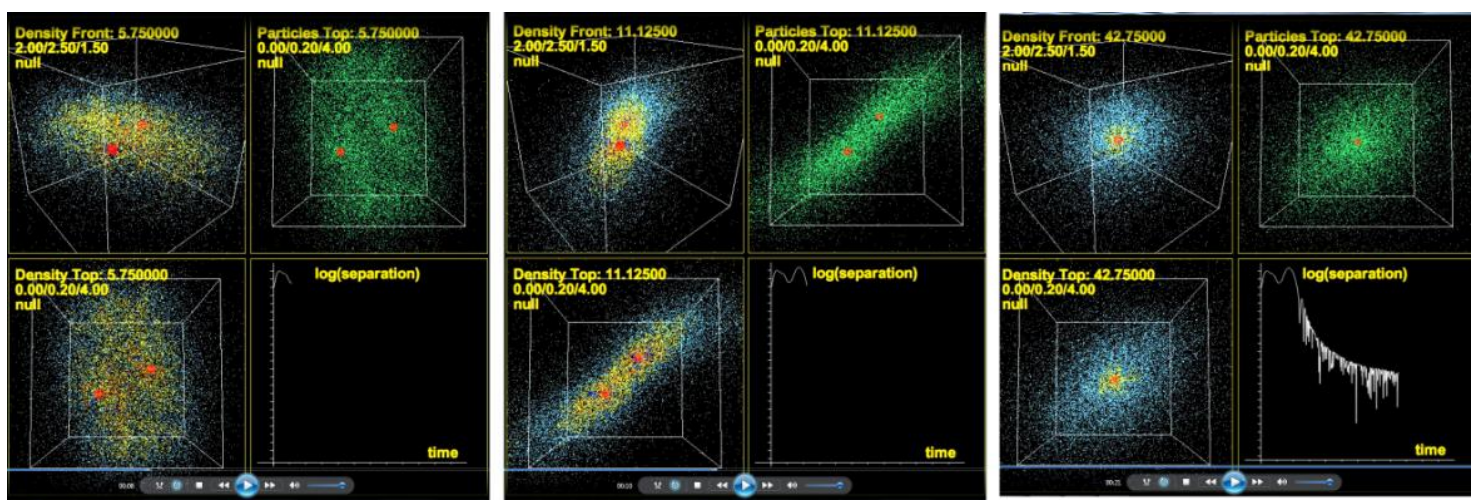

Fig.1 Snapshots of galaxy during the evolution, in each snapshot galaxy from three viewpoints is pictured with the separation of binary Black holes.Positions of Black Holes are indicated with large red circles. The formation of bar instability that gives large elipticities, is clearly visible in second snapshot, later the bar instability dissolves.

In Fig.1 snapshots of movie of evolution of galaxy, is presented to give a clue on evolution picture. Central black holes are given as red circles, the size of circles are large to indicate clearly their position inside the galaxy. As one can see, galaxy evolves to an elongated ellipsoidal shape in the beginning, and then looses the strong elipticity. The movie of evolution of galaxy, is attached.

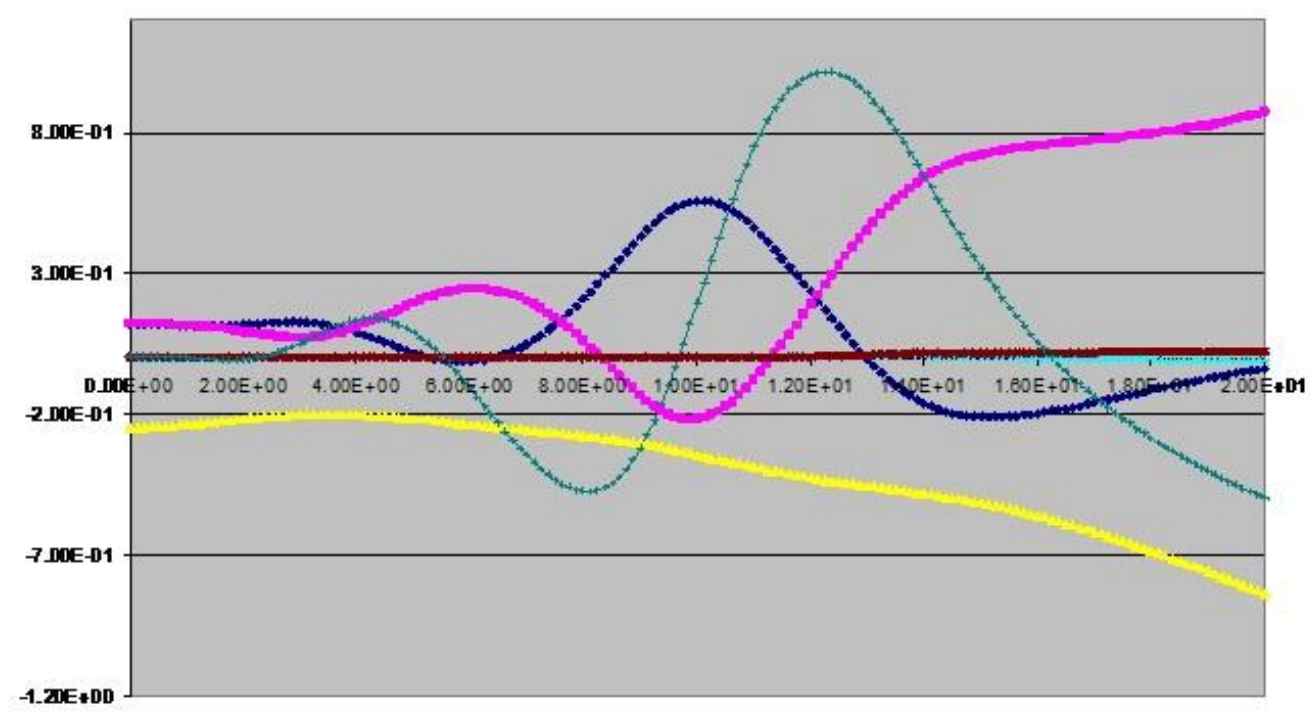

Fig.2 Evolution of components of quadruple moment of galaxy in the beginning of simulation. 
For calculation of gravitational wave radiation main parameters the method described in [7] is used. Although, as it is mentioned in [8] this method is not giving exact values for gravitational wave radiation parameters, but rather upper limits for them, the method is attractive for its simplicity and the flexibility to be used for different types of isolated gravitational wave sources, starting from white dwarfs and ending with galaxies. According to this methodology gravitational wave frequencies are calculated for the whole timescale of evolution, changing in the range from $10^{-13}$ to $10^{-9} \mathrm{~Hz}$.

In Fig. 2. evolution of components of quadruple moment is presented in the beginning of evolution, up to $\mathrm{t}=20$. In blue, red, yellow and magnetta are given correspondingly Qxx, Qyy Qzz and Qxz components of quadruple moment in N-body units that are $9.5 \cdot 10^{86} \mathrm{gcm}^{2}$ vs $3 \cdot 10^{13} \mathrm{~s}$.

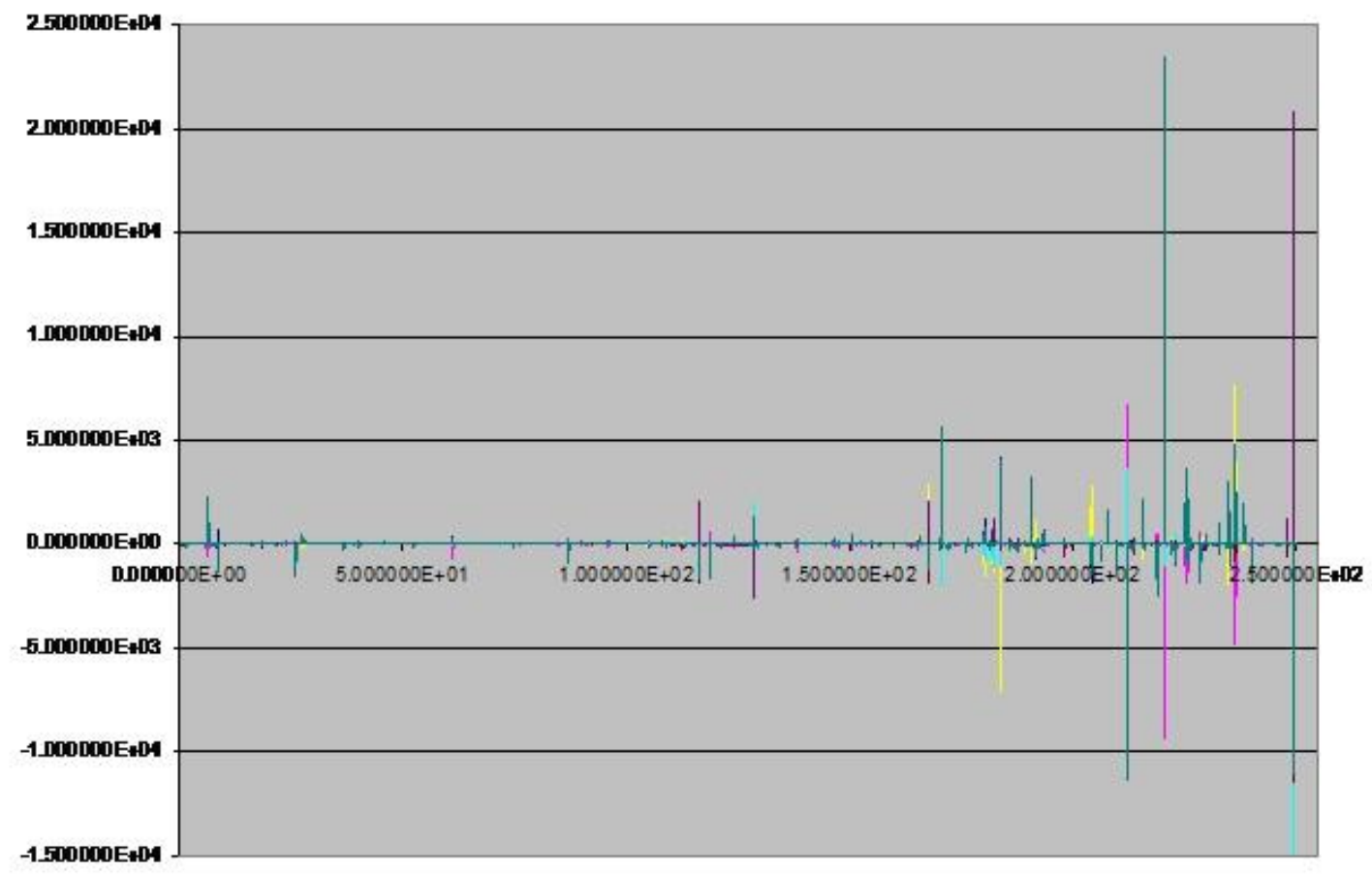

Fig. 3 Spikes in energy flux emitted by the galaxy through gravitational window in different directions. Spikes are becoming stronger to the end of simulation.

In Fig. 3 Overall intensity of gravitational wave radiation from galaxy in all frequency domain is given. We had calculated the total energy flax emitted from the galaxy through gravitational window(Gravitational Luminosity). As one can see from Fig. 3, unexpected spikes in GW radiation with maximum $10^{4}$ over the average are occurring. Colors indicate different directions of gravitational wave radiation. Spikes are becoming more intense with hardening of central binary. 
Fig. 3 is made for the galaxy with 25000 elements, while the existence of spikes persist for all simulated galaxies, picture changes quantitatively, but not qualitatively, regardless to the N-body number. For the discussion on origin of spikes we send the reader to the next section.

On Fig. 4 Comparison of evolution of GW radiation amplitudes on characteristic distance from the source are given for Galaxy and Central engine in N-body units. Both gravitational wave radiations are non monochromatic, frequencies are in Ultra-low frequency band, calculated according [8], changing for galaxy in the range of $10^{-13}$ to $10^{-10} \mathrm{~s}^{-1}$. Gravitational radiation of a central engine is given in red and amplitudes for GW radiation from galaxy is in blue. At the initial phase of radiation GW from galaxy is stronger than GW from central engine. That's because of a "bar" instability formed in galaxy. Later the instability disappears, while the central engine hardens. On the upper left part of Fig. 3 an enlarged scaled part of the same graph is presented for the time period $[0,25]$, to give a better understanding on evolution of gravitational wave radiation from galaxy and from binary in the initial phase of evolution.

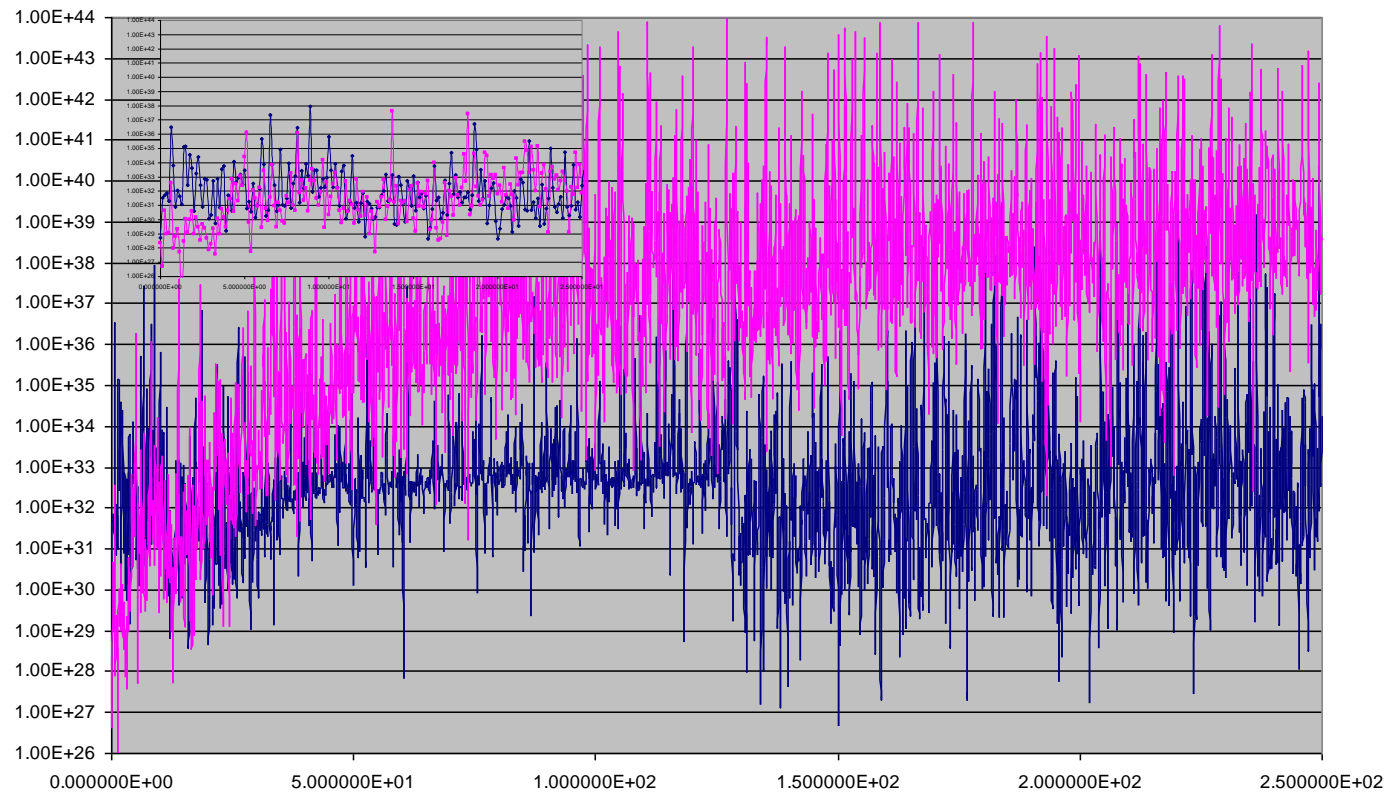

Fig 4. Gravitational wave amplitudes in N-body units on Characteristic distance from galaxy in blue and from binary black hole in red. At initial phases radiation from Galaxy is stronger than from black hole binary.

Different models of galaxies with different initial values are investigated. The most promising results, as it could be expected in advance, are obtained for fast rotating galaxies, where velocities are close to Keplerian. In this galaxies the formation of bar 
instabilities-structures with large ellipticities are formed in the beginning of the evolution, later they are dissolved. As one can see from Fig. 3 there are stages in the beginning of the evolution of the fast rotating galaxies, where the gravitational radiation from galaxy itself due to the bar instability is competing, and in some phases is even stronger than the gravitational radiation of central engine. So we are concluding that for initial stages of galaxy evolution, gravitational radiation from galaxy in ultralow frequency band is as important as the radiation from central binary system and should not be neglected.

\section{Discussions}

We are concentrated in this work on gravitational radiation from galaxy itself, showing that the radiation from galaxy is not neglectable and can be registered in the scope of Parks Pulsar Timing Array project. Although gravitational radiation from coalescing supermassive black holes is strong, it can be expected only at the late stages of galaxy evolution, while we speculate that the majority of galaxies could be found in the stage described by our simulations, i.e. in the beginning of evolutionary path. In this stage of evolution rotating galaxies can develop "bar instabilities", when galaxies take the form of elongated ellipsoids, gaining significantly large quadrupole moments, around $10^{86} \mathrm{gcm}^{2}$, hence they can be considered as interesting sources of gravitational radiation in ultralow frequency band. The gravitational radiation from galaxies is lasting Giga years, while chirp from close binary is maximum several seconds. We had calculated the total energy flax from the galaxy through gravitational radiation (Gravitational Luminosity) and found large energy spikes in Gravitational Luminosity of Galaxies, largest spikes are observed in the time period near to the end of evolution. This spikes in gravitational luminosity of galaxy may be produced by stars that are kicked out by the central binary. "Kicked" stars may have very large velocities [5] enough to leave the galaxy, and we speculate that when they pass the region near to perihelion of their orbit, a spike in gravitational wave radiation energy is occurring. The GW radiation spike is not conditioned by the expansiveness of the quadruple moment, but rather by the "rapid" change of quadruple moment of the given star in short time period. We will double-check the speculation in upcoming calculations. If confirmed experimentally, the existence of spikes in gravitational wave luminosities can be a direct evidence of existence of stars ejected from the galaxies by central engines. Our result concerning the observation of largest spikes near to the end of simulation we explain by the choice of our initial data: where there are no any high elliptical trajectories, this trajectories are formed during the evolution, in other words central engine requires time 
to change the initially semicircular trajectory of a star to highly elliptical, to kick out it from the region of simulation.

Naturally, the question of inner structure of ejected stars accelerated by central engine to velocities high enough to leave the galaxy, is not considered in the scope of $\mathrm{N}$-body problem, which might put additional limitation on the existence of such accelerated stars.

\section{Acknowledgements}

One of authors, AS, acknowledges the DAAD research grant for the visit to Heidelberg Astronomical Rechen Institute.

\section{References}

[1] Lagoute, C \&Longaretti, P-Y, A\&A 308, 441, 1996

[2] King I.R. J. $A J$ 71, 64,1966

[3] Berczik, P. Merrit, D. \&Spurzem, R. ApJ 633, 680, 2005

[4] J. Makino et all, “Grape 6:Massive-parallel Special Purpose Computer for astrophysical Particle Simulations”, PASJ: Publ. Astron. Soc. Japan 55, 1163-1187,2003 December 25

[5] Berczik, P. Merrit, D. Spurzem, R, \& Hans-Peter Bischof ApJ, 642:L21-L24, 2006

[6] Makino J. \& Funato Y. ApJ, 602,93, 2004

[7] Benacquista M., Sedrakian D.M., Hairapetyan M.V. ,Shahabasyan K.M, Sadoyan A.A, ApJ 596: L223-L226, 2003

[8] Sadoyan A.A. Astrofizika, v49, p243-250, 2006.

[9] Edwards, R. T., Hobbs, G. B., \& Manchester, R. N. 2006, MNRAS, 372, 1549 